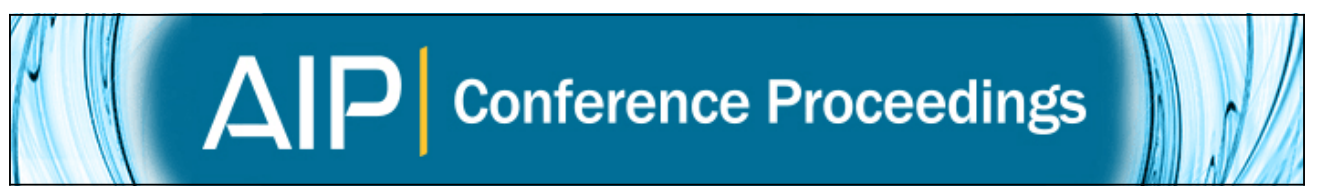

\title{
The LAOG-Planet Imaging Surveys
}

G. Chauvin, A.-M. Lagrange, D. Mouillet, J.-L. Beuzit, H. Beust, D. Ehrenreich, M. Bonnefoy, F. Allard, M. Bessel, M. Bonavita, S. Desidera, C. Dumas, J. Farihi, T. Fusco, D. Gratadour,

P. Lowrance, M. Mayor, D. Rouan, I. Song, S. Udry, and B. Zuckerman

Citation: AIP Conference Proceedings 1158, 183 (2009); doi: 10.1063/1.3215840

View online: http://dx.doi.org/10.1063/1.3215840

View Table of Contents:

http://scitation.aip.org/content/aip/proceeding/aipcp/1158?ver=pdfcov

Published by the AIP Publishing

\section{Articles you may be interested in}

Latest Results from the DODO Survey: Imaging Planets around White Dwarfs

AIP Conf. Proc. 1331, 271 (2011); 10.1063/1.3556210

Planets around Extreme Horizontal Branch Stars

AIP Conf. Proc. 1331, 147 (2011); 10.1063/1.3556194

Spherically Symmetric Accretion onto a Supermassive Black Hole Embedded into a Young Starburst Region

AIP Conf. Proc. 1206, 264 (2010); 10.1063/1.3292531

Homogeneous Comparison of Planet Candidates Imaged Directly Until 2008

AIP Conf. Proc. 1158, 231 (2009); 10.1063/1.3215849

Wide-field Imaging Survey of Dust Continuum Emissions at $\lambda=1.1 \mathrm{~mm}$ toward the Chamaeleon and Lupus Regions with AzTEC on ASTE AIP Conf. Proc. 1158, 141 (2009); 10.1063/1.3215822 


\title{
The LAOG-Planet Imaging Surveys
}

\author{
G. Chauvin*, A.-M. Lagrange*, D. Mouillet*, J.-L. Beuzit*, H. Beust*, D. \\ Ehrenreich*, M. Bonnefoy* $^{*}$ F. Allard ${ }^{\dagger}$, M. Bessel**, M. Bonavita ${ }^{\ddagger}$, S. \\ Desidera $^{\ddagger}$, C. Dumas ${ }^{\S}$, J. Farihi i, T. Fuscoll, D. Gratadour ${ }^{\dagger \dagger}$, P. Lowrance ${ }^{\ddagger \ddagger}$, \\ M. Mayor ${ }^{\S \S}$, D. Rouan ${ }^{\dagger \dagger}$, I. Song ${ }^{\Uparrow \uparrow}$, S. Udry ${ }^{\S \S}$ and B. Zuckerman*** \\ ${ }^{*}$ Laboratoire d'Astrophysique, Observatoire de Grenoble, UJF, CNRS: 414, Rue de la piscine, \\ 38400 Saint-Martin d'Hères, France \\ ${ }^{\dagger}$ Centre de Recherche Astronomique de Lyon, 46 allée d'Italie, 69364 Lyon cedex 7, France \\ ${ }^{* *}$ Research School of Astronomy and Astrophysics Institute of Advance Studies, Australian \\ National University: Cotter Road, Weston Creek, Canberra, ACT 2611, Australia \\ $\$$ Universita' di Padova, Dipartimento di Astronomia, Vicolo dell'Osservatorio 2, 35122 Padova, \\ Italy \\ ${ }^{\S}$ European Southern Observatory: Casilla 19001, Santiago 19, Chile \\ 'Department of Physics \& Astronomy, University of Leicester, Leicester LE1 7RH, United Kingdom \\ Office National d'Etudes et de Recherches Aérospatiales, 29 avenue de la Division Leclerc, 92322 \\ Châtillon, France \\ †ESIA, Observatoire de Paris, 5 place Jules Janssen, 92195 Meudon, France \\ Spitzer Science Center, IPAC/Caltech: MS 220-6, Pasadena, CA 91125, USA \\ $\$ \S$ Observatoire de Genève, $51 \mathrm{Ch}$. des Maillettes, 1290 Sawverny, Switzerland \\ ף Department of Physics \& Astronomy, University of Georgia, Athens, GA 30602-2451, USA \\ ${ }^{* * *}$ Department of Physics \& Astronomy and Center for Astrobiology, University of California: Los \\ Angeles, Box 951562, CA 90095, USA
}

\begin{abstract}
With the development of high-contrast imaging techniques and infrared detectors, vast efforts have been devoted during the past decade to detect and characterize lighter, cooler and closer companions to nearby stars, and ultimately image new planetary systems. Complementary to other observing techniques (radial velocity (RV), transit, micro-lensing, pulsar-timing), this approach has opened a new astrophysical window to study the physical properties and the formation mechanisms of brown dwarfs and planets. Here, I will briefly present the observing challenges, the different observing techniques, strategies and samples of current exoplanet imaging searches that have been selected in the context of the LAOG-Planet Imaging Surveys. Finally, I will describe the most recent results that led to the discovery of giant planets that probably formed like those in our solar system, offering exciting and attractive perspectives for the future generation of deep imaging instruments.
\end{abstract}

Keywords: Instrumentation: adaptive optics, high angular resolution - Methods: observational, data analysis, statistical - Techniques: photometric, astrometric - Stars: low-mass, brown dwarfs, planetary systems>

PACS: $97.82 .-j$

\section{SEARCHING FOR PLANETS}

The search for planets has been an important driver for observers in the two last decades. Their detection and characterization contribute to developing our understanding of their structure, formation and evolution. In the close $(\leq 5 \mathrm{AU})$ environment of stars, the radial velocity, transit, micro-lensing, pulsar-timing observing techniques are the best-suited ones so far. The radial velocity (RV) and transit techniques are currently the most suc-

CP1158, Exoplanets and Disks: Their Formation and Diversity,

Proceedings of the Intemational Conference, edited by T. Usuda, M. Ishii, and M. Tamura

(C) 2009 American Institute of Physics 978-0-7354-0695-7/09/\$25.00 
cessful methods for detecting and characterizing the properties of exoplanetary systems. The RV surveys have focused on main sequence solar-type stars, with numerous narrow optical lines and low activity, to ensure high RV precision. Recently, planet-search programs have been extended to lower- and higher-mass stars $[13,25]$ and younger and more evolved systems $[17,18]$. Since the discovery of 51 Peg b [28], more than 300 exoplanets have been identified featuring a broad range of physical (mass) and orbital $(\mathrm{P}, e)$ characteristics $[34,2]$. This technique also revealed the existence of the so-called brown dwarf desert at small ( $\leq 5 \mathrm{AU}$ ) separations [15]. The bimodal aspect of the secondary mass distribution indicates different formation mechanisms for two populations of substellar companions, brown dwarfs and planets. The transit technique coupled with RV enables determination of the radius and density of giant planets, and thus is a probe of their internal structure. Moreover, spectral elements of a planetary atmosphere can be revealed during primary or secondary eclipse $[31,16]$. To extend such systematic characterization at larger scales ( $\geq 10 \mathrm{AU}$ ), the deep imaging technique is particularly wellsuited to probe the existence of planets and brown dwarf companions and complete our view of planetary formation and evolution. To access small angular separations, the $H u b$ ble Space Telescope or a combination of adaptive optics (AO) systems with very large ground-based telescopes (Palomar, Canada-France-Hawai'i Telescope (CFHT), Keck, Gemini, Subaru, Very Large Telescope) have become mandatory. We present here the results of the three main planet-search surveys conducted at the Grenoble Institute of Astrophysics (LAOG) since 2002.

\section{THE ENVIRONMENT OF EXOPLANET HOST STARS}

We have conducted a deep coronographic AO imaging survey of 26 stars with planets detected through radial velocity measurements [8]. The domain investigated typically ranges between $0.1^{\prime \prime}$ to $15^{\prime \prime}$ (i.e. about 3 to $500 \mathrm{AU}$, according to the mean distance of the sample). The survey is sensitive to stellar and substellar companions with masses greater than $30 \mathrm{M}_{\text {Jup }}(0.5 \mathrm{Gyr})$ with CFHT and $15 \mathrm{M}_{\text {Jup }}(0.5 \mathrm{Gyr})$ with VLT, at 2 " $(\sim 60 \mathrm{AU})$ from the primary star. Among the 20 stars that were found to have potential companions (candidate companions, hereafter CCs), we could identify (through proper motion measurements) and then monitor three bound companions to three stars: 1) HD1237 is orbited by a $0.13 \mathrm{M}_{\odot}$ star at about $70 \mathrm{AU}$ (proj. distance) with an orbital motion marginally resolved; 2) HD 27442 is orbited by a white dwarf located at 240 AU. This is the third white dwarf discovered so far around a star that is also hosting planets, in addition to G186 B and HD 147513 B [30]. In both cases, the RV drift induced by these companions are below the limits of current RV studies. This illustrates the complementarity between direct imaging and RV studies. Finally, we also found a 0.55 $\mathrm{M}_{\odot}$ star (assuming an age of $0.5 \mathrm{Gyr}$ ) orbiting HD196885. This latter case brings to five the number of stars hosting planets and members of close-in ( $\leq 20 \mathrm{AU}$ ) multiple systems. Combining $\mathrm{AO}$ and $\mathrm{RV}$ data, we were able to already strongly constrain the mass and orbit of both systems: G186 B $\left(0.48-0.54 \mathrm{M}_{\odot} ; e \geq 0.4 ; a=18 \mathrm{AU} ;[23]\right.$ and Fig. 1) and $\mathrm{HD} 196885 \mathrm{~B}\left(\mathrm{M}=0.55 \mathrm{M}_{\odot} ; e=0.45 ; a=26 ;\right.$ [9]). We additionaly traced back the evoluttionary and dynamical history of G186 B, which was originally a G dwarf on a close $\left(a_{i n i}=13 \mathrm{AU}\right.$; ) and less eccentric orbit. Several open questions remain related 

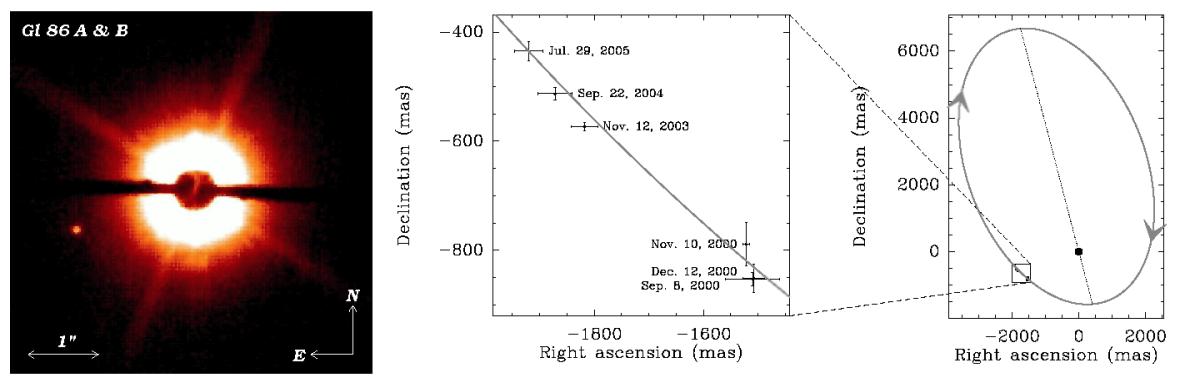

FIGURE 1. Left: A VLT/NACO Ks band coronagraphic image of GLiese $86 \mathrm{~A}$ and B, acquired on September 24, 2004, with an occulting mask of diameter 0.7". Right: A representation of the orbital solutions, as projected onto the plane of the sky (view of the full orbit and an enlargement).

to the fact that the exoplanet must have survived all the late evolution stages of G186 B.

\section{PROBING PLANETS ORBITING DUSTY STARS}

The dusty star survey was focused on probing the presence of planets around about 15 stars with known debris disks - disks containing dust which is not primordial but produced by collisions among larger rocky bodies. In the course of this survey, a companion candidate was detected around $\beta$ Pic, a A5V star at a distance of $19.3 \pm 0.2 \mathrm{pc}$ [11], which remains the best-studied young ( $12_{-4}^{+8} \mathrm{Myr}$; [35]) system, with an impressive amount of indirect signs pointing toward the presence of planets.

The disk shows a relative inner void of matter inside 50 AU. Lecavelier DEs Etangs et al. [26] presented intriguing light variations possibly due to disk inhomogeneities produced by a Jupiter-size planet at $>6$ AU. Several asymmetries have been identified in the disk at optical $[20,19]$ and infrared [32] wavelengths, as well as a warp at $\sim 50 \mathrm{AU}$ $[29,19]$. The structure is well reproduced by the deformation induced on colliding planetesimals by a giant planet on a slightly inclined orbit within $50 \mathrm{AU}$ from the star $[22,29,14,1,33]$. Using deep adaptive optics $L^{\prime}$-band images, a faint point-like signal is detected at a projected distance of $\simeq 8 \mathrm{AU}$ from the star, within the northeast side of the dust disk (see Fig. 2). Various tests were made to rule out possible instrumental or atmospheric artefacts with a good confidence level. The probability of a foreground or background contaminant is extremely low, based on the analysis of previous deep $H S T$ images. Its $L^{\prime}=11.2$ apparent magnitude would indicate a typical temperature of $\sim 1500 \mathrm{~K}$ and a mass of $\sim 8 \mathrm{M}_{\text {Jup }}$. If confirmed, it could explain the main morphological and dynamical peculiarities of the $\beta$ Pic system. During the same year, Marois et al. [27] and Kalas et al. [21] have reported images of giant planets to intermediate-mass stars. The present detection remains unique by the proximity of the resolved planet. Its closeness and location inside the $\beta$ Pic disk suggest a formation process by core accretion or disk instabilities rather than binary like formation processes. 

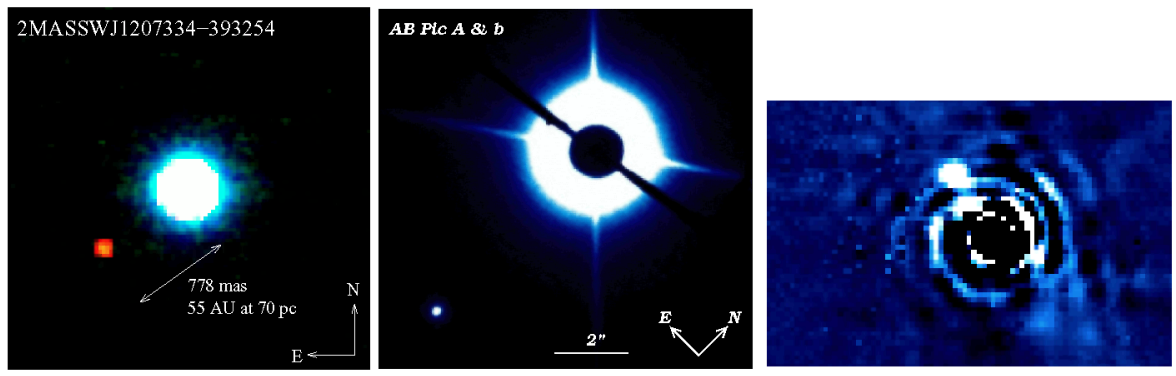

FIGURE 2. Left: VLT/NACO deep image of the TWA brown dwarf $2 \mathrm{M} 1207$ and its $5 \mathrm{M}_{\text {Jup }}$ companion at $41 \mathrm{AU}$ obtained in $K_{s}$-band with the S27 camera (Chauvin et al. 2004). Middle: VLT/NACO coronagraphic imaging with the small $\left(\varnothing=0.7^{\prime \prime}\right)$ coronagraphic mask of the $13 \mathrm{M}_{\text {Jup }}$ companion to the $A B$ Pic star, member of the Tuc-Hor association [6]. Right: VLT/NACO deep L' image of the $\beta$ Pictoris b candidate detected thanks to an optimized PSF-reference subtraction.

\section{YOUNG, NEARBY AUSTRAL STARS}

Finally, we have conducted a deep adaptive optics imaging survey with NACO at the VLT of 88 nearby stars in the southern hemisphere [10]. Our selection criteria favored youth $(\leq 100 \mathrm{Myr})$ and proximity to Earth $(\leq 100 \mathrm{pc})$ to optimize the detection of close planetary mass companions. Known visual binaries were excluded to avoid degrading the NACO AO and/or coronagraphic detection performance. Among our sample, 51 stars are members of young, nearby co-moving groups. We found 32 are young, nearby stars currently not identified as members of any currently known association and five have been reclassified as older ( $\geq 100 \mathrm{Myr}$ ) systems. The spectral types cover the sequence from B to $\mathrm{M}$ spectral types with $19 \%$ BAF stars, $48 \% \mathrm{GK}$ stars and $33 \% \mathrm{M}$ dwarfs. The separation investigated typically ranges between $0.1^{\prime \prime}$ to $10^{\prime \prime}$, i.e. generally between 10 to $500 \mathrm{AU}$. A sample of 65 stars was observed in deep coronagraphic imaging to enhance our contrast performances to $10^{-6}$ and to be sensitive to planetary mass companions down to $1 \mathrm{M}_{\text {Jup }}$ (at $24 \%$ of our sample) and $3 \mathrm{M}_{\text {Jup }}$ (at $67 \%$ ). We used a standard observing sequence to measure precisely the positions and the flux of all detected sources relative to their visual primary stars. Repeated observations at several epochs enabled us to discriminate co-moving companions from background objects. In the course of that survey, we discovered 17 new close $\left(0.1-5.0^{\prime \prime}\right)$ multiple systems. HIP $108195 \mathrm{AB}$ and C (F1III-M6), HIP $84642 \mathrm{AB}(a \sim 14 \mathrm{AU}, \mathrm{K} 0-\mathrm{M} 5)$ and TWA22 AB $(a \sim 1.8 \mathrm{AU}$; M6-M6) are confirmed as co-moving systems. TWA22 AB, with $80 \%$ of its orbit already resolved, is likely to be a rare astrometric calibrator for testing evolutionary model predictions. About 236 faint CCs were detected around 36 stars observed in coronagraphy. Follow-up observations with VLT or HST for 30 stars enabled us to identify their status. $1 \%$ of the CCs detected have been confirmed as comoving companions, $43 \%$ have been identified as probable background contaminants and about $56 \%$ need further follow-up observations. The remaining CCs come mostly from the presence of crowded fields in the background of the 6 stars observed at one epoch. We confirmed previously discovered substellar companions around GSC 08047- 

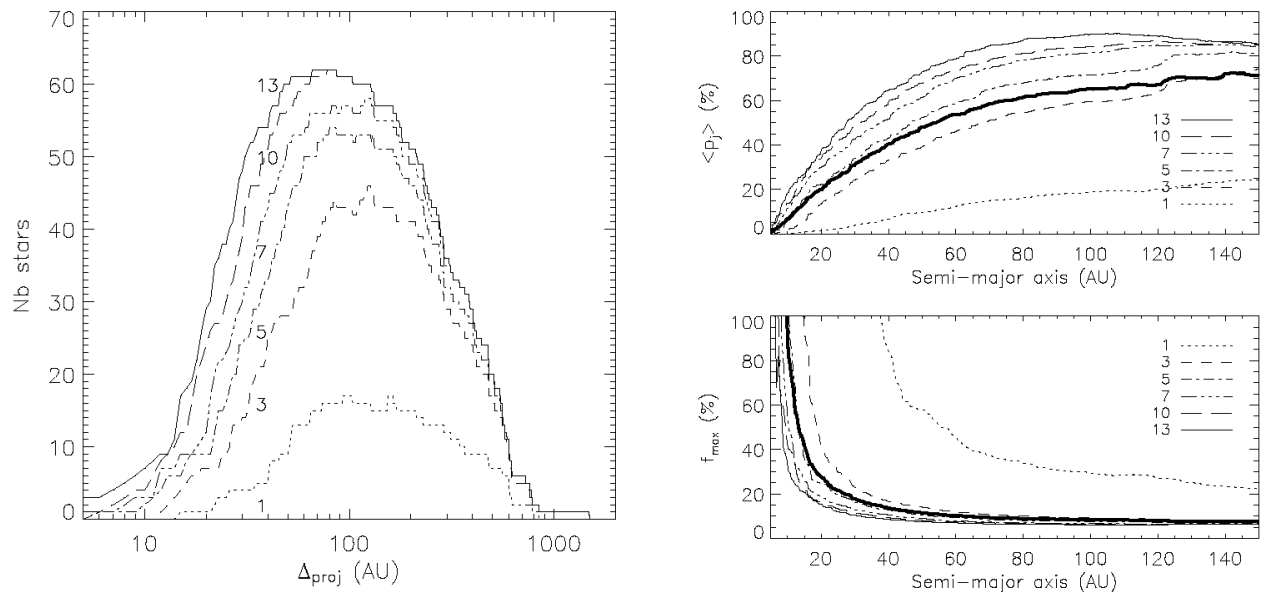

FIGURE 3. Left: a histogram of projected physical separations explored, for various planetary masses $(1,3,5,7,10$ and 13$) \mathrm{M}_{\mathrm{Jup}}$, in the close vicinity of the 65 young, nearby stars observed with NACO at VLT in coronagraphy [10]. Contrast performances have been converted into masses based on the near-infrared photometry, age and distance of the primary stars. Right:, Top: survey mean detection probability derived as a function of semi-major axis assuming parametric mass and period distributions derived by [12], i.e with $\alpha=-1.31, \beta=-0.74$ and $\gamma=1.25$. The results are reported for individual masses: $1,3,5,7,10$ and $13 \mathrm{M}_{\mathrm{Jup}}$. The integrated probability for the planetary mass regime is shown with the thick solid line. Bottom: planet fraction upper limit derived as a function of semi-major axis, given the same mass and period distributions.

$00232[3,5], \mathrm{AB} P i c[6]$ and $2 \mathrm{M} 1207$ [4, 5] and placed them in the perspective of confirmed substellar companions among young, nearby associations (see Fig. 3). Finally, the statistical analysis of our complete set of detection limits enables us to constrain at large semi-major axes, 20 to a few 100 AU, various mass, period and eccentricity distributions of giant planets extrapolated and normalized from RV surveys. It enables us to derive limits on the occurrence of giant planets for a given set of physical and orbital distributions. The survey starts significantly constraining the population of giant planet for masses $\geq 3$ Mup (see Fig. 4).

\section{CONCLUSION AND PERSPECTIVE}

In the first few years following the discovery of the companion to $2 \mathrm{M} 1207$ [4], all planetary mass companions were discovered at relatively wide separations or with a small mass ratio with their primaries. However, the recent discoveries of planetary-mass objects around the star Fomalhaut [21], HR 8799 [27] and $\beta$ Pictoris [24], now open a new era for the deep imaging study of giant planets that probably formed like those of our solar system. In the perspective of ongoing and future deep imaging instruments either from the ground (Gemini/NICI, Subaru/HiCIAO, SPHERE, GPI, EPICS) or from space ((JWST, TPF/Darwin), this work represents a successful pioneering study providing, 
along with other surveys, precise information (stellar and substellar multiplicity, nondetections and background contaminants) to better characterize the overall environment of young, nearby stars, that will be prime targets for futur exoplanets search.

\section{ACKNOWLEDGMENTS}

We thank all the organizers (LOC and SOC) of the 2nd Subaru International Conference "Disks to Exoplanets." For all results presented, we thank the ESO Paranal staff for performing the service mode observations. We also acknowledge partial financial support from the PNPS and Agence National de la Recherche, in France, from INAF through PRIN 2006 "From disk to planetary systems: understanding the origin and demographics of solar and extrasolar planetary systems" and from NASA.

\section{REFERENCES}

1. Augereau, J.-C., Nelson, R. P., Lagrange, A.-M. et al. 2001, A\&A, 370, 447

2. Butler R. P., Wright J.T., Marcy G.W. et al. 2006, ApJ, 646, 505

3. Chauvin G., Thomson M., Dumas C. et al. 2003, A\&A, 404, 157

4. Chauvin G., Lagrange A.-M., Dumas C. et al. 2004, A\&A, 425, L25

5. Chauvin G., Lagrange A.-M., Lacombe F. et al. 2005, A\&A, 430, 1027

6. Chauvin G., Lagrange A.-M., Dumas C. et al,, 2005, A\&A, 438, L25

7. Chauvin G., Lagrange A.-M., Zuckerman B. et al. 2005, A\&A, 438, L29

8. Chauvin G., Lagrange A.-M., Udry S. et al. 2006, A\&A, 456, 1165

9. Chauvin G., Lagrange A.-M., Udry S. et al. 2007, A\&A, 475, 723

10. Chauvin G., Lagrange A.-M., Bonavita M. et al. 2009, A\&A, accepted

11. Crifo, F., Vidal-Madjar, A., Lallement, R., Ferlet, R., \& Gerbaldi, M. 1997, A\&A, 320, L29

12. Cumming A., Butler R. P., Marcy G. W. et al. 2008, PASP, 120, 531

13. Endl M., Cochran W.D., KÃijrster M. et al. 2006, ApJ, 649, 436

14. Gorkavyi, N., Heap, S., Ozernoy, L., Taidakova, T., Mather, J. 2000, astro.ph.12470

15. Grether \& Lineweaver 2006, ApJ, 640, 1051

16. Grillmair C.J., Burrows A., Charbonneau D. et al. 2008, Nature, 456, 767

17. Joergens V. 2006, A\&A, 446, 1165

18. Johnson J.A., Fischer D.A., Marcy G.W. et al. 2007, ApJ, 665, 785

19. Heap, S. R., Lindler, D. J., Lanz, T. M., et al. 2000, ApJ, 539, 435

20. Kalas, P., \& Jewitt, D. 1995, AJ, 1 10, 794

21. Kalas P., Graham J. R., Chiang E. et al. 2008, Science, 322, 1345

22. Krist, J.E., Burrows, C.J., Stapelfeldt, K.R., Watson, A.M.,\& WFPC2 Investigation Definition Team 1995, AAS, 187, 4413

23. Lagrange A.-M., Beust H., Udry S. et al. 2006, A\&A, 459, 955

24. Lagrange A.-M., Gratadour D., Chauvin G. et al. 2008, A\&A, 493, L21

25. Lagrange A.-M., Desort M., Galland F., Udry S. \& Mayor M. 2009, A\&A, 495, 335

26. Lecavelier Des Etangs, A.; Deleuil, M.; Vidal-Madjar, A. et al. 1995, A\&A, 299, 557

27. Marois C., Macintosh B., Barman, T. et al. 2008b, arXiv0811.2606

28. Mayor, M. \& Queloz, D. 1995, Nature 378, 355

29. Mouillet, D., Larwood, J. D., Papaloizou, J. C. B., \& Lagrange, A.-M. 1997, MNRAS, 292, 896

30. Porto de Mello G. F. \& da Silva L. 1997, ApJ, 476, 89

31. Swain M.R., Vasisht G. \& Tinetti G. 2008, Nature, 452, 329

32. Telesco, C. M., Fisher, R. S., Wyatt, M. C., et al. 2005, Nature, 433, 133

33. Thébault, P. \& Beust, H. 2001, A\&A, 376, 621

34. Udry S. \& Santos N. C. 2007, ARA\&A, 45, 397

35. Zuckerman, B., Song, I., Bessel, M. S., \& Webb, R. A. 2001, ApJ, 562, L87 